\title{
Mining actionable information from security forums: the case of malicious IP addresses
}

\author{
Joobin Gharibshah*, Tai Ching Li*, Andre Castro*, \\ Konstantinos Pelechrinis ${ }^{\dagger}$, Evangelos E. Papalexakis* and Michalis Faloutsos* \\ * University of California - Riverside, CA \\ Email: \{jghar002,tli010,acast050,epapalex,michalis $\} @$ cs.ucr.edu \\ $\dagger$ School of Information Sciences, University of Pittsburgh, Pittsburgh, PA \\ Email: kpele@pitt.edu
}

\begin{abstract}
The goal of this work is to systematically extract information from hacker forums, whose information would be in general described as unstructured: the text of a post is not necessarily following any writing rules. By contrast, many security initiatives and commercial entities are harnessing the readily public information, but they seem to focus on structured sources of information. Here, we focus on the problem of identifying malicious IP addresses, among the IP addresses which are reported in the forums. We develop a method to automate the identification of malicious IP addresses with the design goal of being independent of external sources. A key novelty is that we use a matrix decomposition method to extract latent features of the behavioral information of the users, which we combine with textual information from the related posts. A key design feature of our technique is that it can be readily applied to different language forums, since it does not require a sophisticated Natural Language Processing approach. In particular, our solution only needs a small number of keywords in the new language plus the user's behavior captured by specific features. We also develop a tool to automate the data collection from security forums. Using our tool, we collect approximately $600 \mathrm{~K}$ posts from 3 different forums. Our method exhibits high classification accuracy, while the precision of identifying malicious IP in post is greater than $88 \%$ in all three forums. We argue that our method can provide singificantly more information: we find up to 3 times more potentially malicious IP address compared to the reference blacklist VirusTotal. As the cyber-wars are becoming more intense, having early accesses to useful information becomes more imperative to remove the hackers first-move advantage, and our work is a solid step towards this direction.
\end{abstract} rums

Keywords: Security, Online communities mining, Fo-

\section{INTRODUCTION}

How can we take the first-mover advantage away from hackers? We argue that hacker forums provide information earlier than other sources, and we should leverage these forums in our security intelligence. Here, we focus on a specific question. In particular, we want to extract as much useful information from hacker/security forums as possible in order to perform (possibly early) detection of malicious IP addresses, e.g., prior to their appearance on blacklists. The latter can exhibit large delays in their update and hence, new ways for labeling malicious IP addresses are needed [15]. In this study we will use the term "hacker forums" to describe online forums with a focus on security and system administration. Interestingly, we can classify these forums into categories: (a) main stream forums, like Wilders Security, and (b) "fringe" forums, like Offensive Community, where we find users with names like satan911. Some of these forums have been known to have hackers boast of attacks they have mounted, or sell tools for malicious purposes (think rent-a-botnet). For example, in our dataset there is a post that mentions "I give you a second server to have your fun with. Multiple websites on this server. So let's see if anyone can actually bring down the server". Right after that the hacker posted the IP, username and password for anyone to access the server. In fact, there is a show-off section in these forums for people to broadcast their hacking "skills".

The overarching goal of this work is to mine the unstructured, user-generated content in security forums. Specifically, we focus here on collecting malicious IP addresses, which are often reported at such forums. We use the term security forum to refer to discussion forum with a focus on security, system administration, and or more generally, systems-related discussions. The users in these forums include: security professionals, hobbyists, and hackers, who go on these forums to identify issues, discuss solutions, and in general exchange information.

Let us provide a few examples of how users report IP addresses, which may or may not be malicious. Posts could talk about a benign IP address, say in configuration files, as in the post:

"[T]his thing in my hosts file: 64.91.255.87 ... [is] it correct?".

At the same time, posts could also report compromised or malicious IP addresses, as in the post:

"My browser homepage has been hijacked to http://69.50.191.51/2484/".

The challenge is to automatically distinguish between the two. By doing so, we can provide a new source of information of malicious IP addresses directly from the affected individuals. Formally, we can state the problem as follows:

Key Question: Malicious IP Detection. Given a set of posts $\mathcal{P}_{F}$ that may contain IP addresses and users $\mathcal{U}_{F}$ of a security forum $F$, as well as, the features $\Phi_{p}, \forall p \in \mathcal{P}_{F}$ and $\Phi_{u}, \forall u \in \mathcal{U}_{F}$ for the posts and the users respectively, can we determine if a given IP address $i$ is malicious or not?

The set of features $\mathcal{P}_{F}$ includes attributes such as the text 
of the post, the posting user, the time of post, etc., while $\mathcal{U}_{F}$ includes information such as the date of a user joining the forum, the number of posts the user has made etc. The above problem has two associated questions:

a. Exclusivity: How many IP Addresses can we find that are never reported by other reference sources?

b. Early warning: How much earlier are malicious IP Addresses reported in a forum compared to reference sources, for the IP Addresses reported by both?

Most previous studies in this area have focused on structured information sources, such as security reports, or malware databases. In fact, many efforts focus on addressing security problems using knowledge obtained from the web, as well as social and information networks. These efforts are mainly focused on analyzing structured sources (e.g., [16]). However, studies assessing the usefulness of (unstructured) information in online forums have only recently emerged (e.g., [23]). These studies are rather exploratory and provide evidence of the usefulness of the data in the forums, but do not provide a systematic methodology or ready-to-use tools, which is the goal of our work. We discuss existing literature in more detail later in section $\mathrm{V}$

The motivation of our work is to provide more information to security analysts and systems. We want to enhance and complement, but not replace, existing efforts for detecting malicious IP Addresses. For instance, many IP blacklists enlist an IP as malicious after a number of reports above a predefined threshold have been made for the specific address. Depending on the threshold and the reactivity of the affected users/systems, this might take several days, weeks or months. Therefore, a system, like the one proposed here, can identify and point to malicious IP address to blacklist services and firewalls.

We propose InferIP, a systematic approach for identifying malicious IP Addresses among the IP addresses, which are mentioned in security forums. A key novelty is that we use the behavioral information of the users, in addition to the textual information from the related posts. Specifically, we customize and use a Sparse Matrix Regression method on this expanded set of features.

This paper presents an extension of our previous work [14]. Here, we add some spatiotempral and behavioral analysis to extract the characteristics of the identified IP addresses and the users who used these IP address in their posts. Moreover, we investigate the ability of the proposed method to provide early warning regarding malicious IP addresses.

By design, our framework is applicable to forums in different languages as it relies only on the behavioral patterns of users and simple word counts, and not a complex languagespecific Natural Language Processing technique. From a technical point of view the challenge in designing a solution to our Key Question is most IP Addresses mentioned in these forums are not malicious. We show that our system can add a significant number of previously unreported IP address to existing blacklist services. Finally, as an engineering contribution, we develop a customizable tool to facilitate the crawling of forums, which we discuss in the next section.

Our results can be summarized into the following points: a. Our method exhibits precision and recall greater than $88 \%$ and $85 \%$ respectively, and an accuracy over malicious class above $\mathbf{8 6 \%}$ in the 10 -fold cross validation tests we conducted for the three different forums. In partially answering our Key Question, if our method labels a currently non-blacklisted IP as malicious, there is a high chance that it is malicious, given our high precision.

b. Our method identifies three times more malicious IP Addresses compared to VirusTotal [5] a widely used aggregator of 60 blacklists of IP addresses. Across our three forums, we find more than 2000 potential malicious IP Addresses that were never reported by VirusTotal.

c. Our method identifies more than half of the IP addresses at least 3 month earlier than VirusTotal. We study the malicious IP addresses that are identified by both VirusTotal and InferIP. We find 53\%, 71\% and 62\% of these IP addreses in Wilders Security, Offensive Community and Ashiyane respectively at least 3 months earlier than they were reported in VirusTotal.

d. The number of reported malicious IP addresses has increased by a factor 8 in 4 years. We find that the number of malicious IP addresses has increased from roughly 100 in 2011 and 2012 to more than 800 in 2016. This could be attributed to either an increase in the user base, an increase in the number of attacks, or a combination of the two.

\section{DAta Collection AND BAsic Properties}

We have collected data from three different forums relevant to our study; (i) Wilders Security [6], (ii) Offensive Community [4], (iii) Ashiyane [1]. The first two forums are mainly written in English, while the last forum is an Iranian forum, in Farsi1.

Our data collection tool. We develop a customizable universal tool to make the crawling forums easier. The challenge here is that each forum has its own format and layout. Our tool requires only a custom configuration file, before crawling a new forum. In configuration file, we specify entities in the forum which are needed such as user ID, post's date, post's content and etc by XML Path Language known as Xpath. Leveraging our current configuration files, the task of crawling a new forum is simplified significantly. Using our crawler, we collect data from three forums, two English and one in Farsi for a total number of more than $30 \mathrm{~K}$ users and $600 \mathrm{~K}$ posts.

We use VirusTotal [5] as our reference blacklist IP addresses, since it is an aggregator, and combines the information from over 70 other blacklists and resources. VirusTotal is free to end users for non-commercial use and is a private API to query the services in the rate of more than 4000 IP addresses per minutes. It is provided upon requests for academic purposes.

We provide some basic statistics for our three forums in Table III Offensive Community and Ashiyane are two fringe forums in different languages. In these forums there is a section where people openly boast about their achievement in hacking. They share their ideas and tutorials on how to break into

${ }^{1}$ Our software and datasets will be made available at:
https://github.com/hackerchater/


TABLE I: Extracting useful information; Number of malicious IP Addresses found by InferIP and not by VirusTotal.

\begin{tabular}{c|c|c|c}
\hline \multicolumn{2}{l|}{} & \multicolumn{2}{c}{ IP found by } \\
\hline Dataset & Total IP & Virus Total & InferIP only \\
\hline Wilders Security & 4338 & 216 & $\mathbf{6 7 0}$ \\
Offensive Community & 7850 & 339 & $\mathbf{6 1 7}$ \\
Ashiyane & 8121 & 133 & $\mathbf{8 0 6}$ \\
\hline
\end{tabular}

vulnerable networks. On the other hand, Wilders Security as a mainstream forum is mostly used to protect non-experts against attacks such as browser hijacking, and provide solutions for their security problems.

For completeness, we present some of the terms we use here. A user is defined by a login name registered with the site. The term post refers to a single unit of content generated by a user. A thread refers to a collection of posts that are replies to a given initiating post.

In Figures 1 and 2, we present the cumulative complementary distribution function of the number of posts per user and the number of threads per users respectively. As we can see in all the cases the distributions are skewed, that is, most of the users contribute few posts in the forums and engage with few threads. In Wilders Security, $85 \%$ of users post less than 10 posts each, while $5.2 \%$ of the users post more than 50 posts. We find that $70 \%$ of the users post in only one thread and only $8 \%$ of the users are active in more than 10 threads. This skewed behavior is typical for online users and communities [12]. We will use features to capture aspects of both these user properties, as we will see in the next section.

In Figure 3, we present the cumulative complementary distribution function of the number of IP addresses that appear in each post. The skewed distribution shows that most of the posts contain a few number of IP address. We find that $84.2 \%$ of the posts with IP addresses in Wilders Security and $84.1 \%$ in Offensive Community have two or less IP addresses. In Ashiyane, $87.2 \%$ of these posts contain less than two IP addresses. Interestingly, in Ashiyane, we find $1 \%$ of the IP containing posts with more than 100 IP addresses. We investigated and we found that typically, these posts provide benign IP addresses of proxies servers to fellow administrators.

Groundtruth for training and testing. In order to build and evaluate, our model we need to obtain a reasonably labeled dataset from IP addresses that appear in the posts of the security forums. For that, we use the VirusTotal service and assign malicious labels to an IP that has been reported by this service. The number of malicious IP Addresses that we have used with the corresponding posts are shown in table $\Pi$ as the IP found by VirusTotal. Note that the absence of a report on VirusTotal does not necessarily mean that the IP is benign. However, a listed IP address is most likely malicious, since VirusTotal as most blacklist sites require a high threshold of confidence for blacklisting an address. This way, we find in total 688 malicious IP addresses for our forums as shown in Table [

Using this labeling process we have collected all the IP addresses that have appeared on our forums prior to their report on VirusTotal. For building our model, we also randomly select an equal number of IP addresses that have not been reported as malicious and via manual inspection further assess their status. Finally, for every security forum we have a different dataset and hence, we build a different model.

\section{INFERIP: MALICIOUS IP DETECTION}

We propose a method to identify whether an IP address within a post is malicious. For example, although many users report a malicious IP address, such as one that is attacking the user's network, there are also users that will mention a benign IP address when people discuss about network tutorials like setting up Putty or initiating a $S S H$ connection.

While this task is simple for a human, it is non-trivial to automate. Adding to the challenge, different communities use different terminology and even different languages altogether (english and farsi in our case). In order to overcome these challenges, we use a diverse set of features and build a model to identify IP addresses that are potentially malicious.

Our approach consists of four steps that each hide nontrivial novelties:

Step 1: We consider the user behavior and extract features that profile users that post IP-reporting posts.

Step 2: We extract keywords from the posts and use information gain to identify the 100 most informative features.

Step 3: We identify meaningful latent feature sets using an unsupervised co-clustering approach [19].

Step 4: We train a classifier using these latent feature sets using 10-fold cross validation.

We describe each step in more detail.

Step 1: Behavioral Features. We associate each user of the forum with a set of 11 features that capture their behavior. In particular:

- Number of posts; the total number of posts made by the user

- Number of threads; the total number of threads the user has contributed to

- Number of threads initiated; the total number of threads initiated by the user

- Average thread entropy; the average entropy of the user distribution of the threads in which the user has contributed to

- Number of active days; the number of days that the user generates at least one post 
TABLE II: The collected forums.

\begin{tabular}{|c|c|c|c|c|}
\hline Forum & Threads & Posts & Users & Active days \\
\hline Wilders Security & 28661 & 302710 & 14836 & 5227 \\
\hline Offensive Comm. & 3542 & 25538 & 5549 & 1508 \\
\hline Ashiyane & 67004 & 279309 & 22698 & 4978 \\
\hline
\end{tabular}

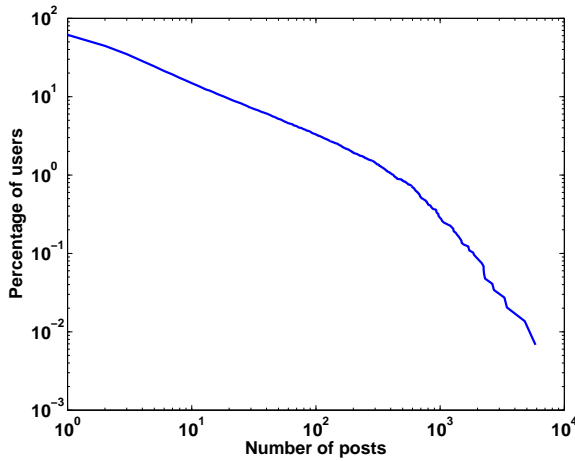

(a) Wilders Security.

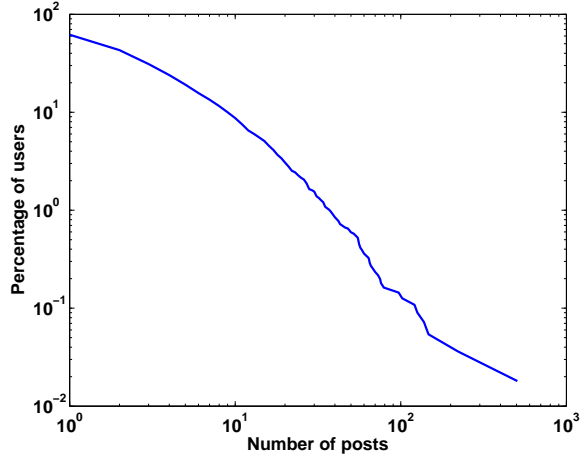

(b) Offensive Community.

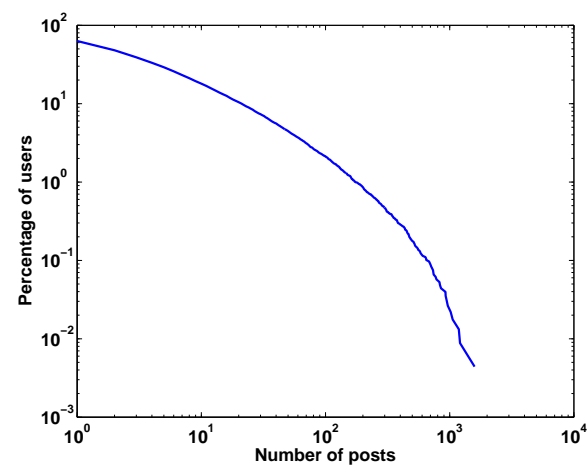

(c) Ashiyane

Fig. 1: CCDF of the number of posts per user (log-log scale).

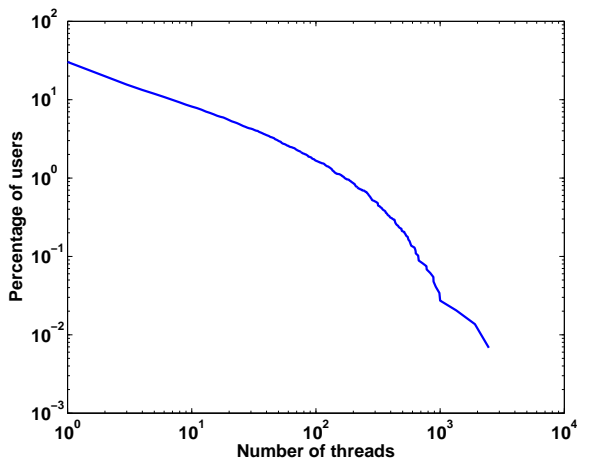

(a) Wilders Security

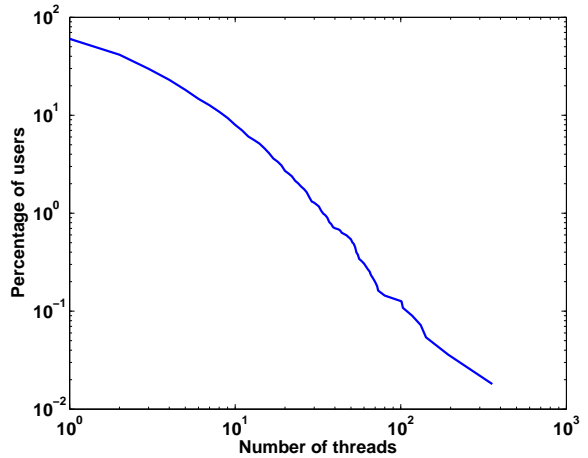

(b) Offensive Community

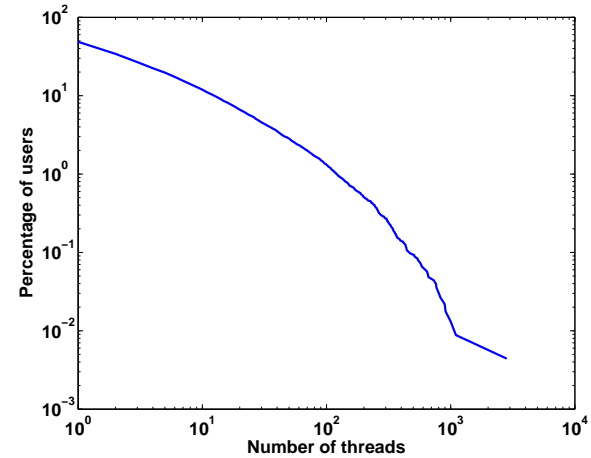

(c) Ashiyane

Fig. 2: CCDF of the number of thread per user (log-log scale).

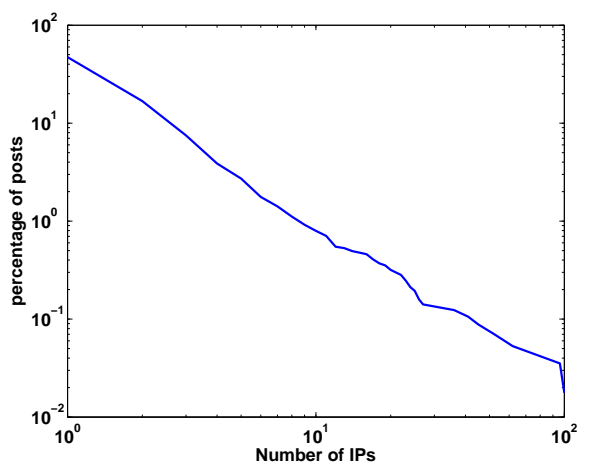

(a) Wilders Security

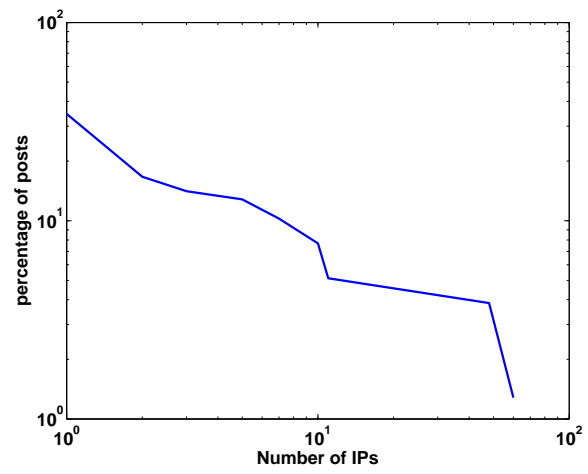

(b) Offensive Community

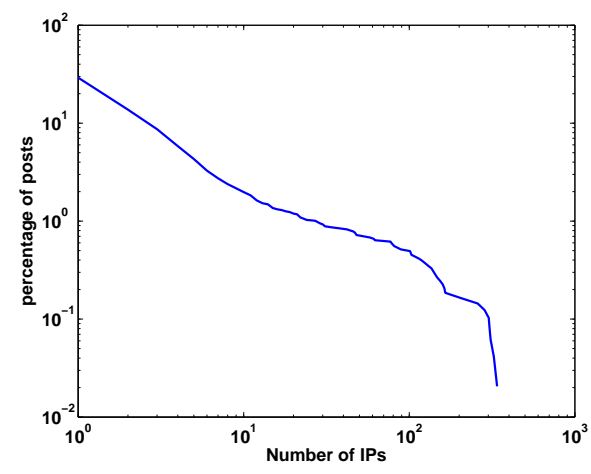

(c) Ashiyane

Fig. 3: CCDF of the number of IP addresses per post (log-log scale).

- Average day entropy; the average entropy of the user distribution of the posts made on the days that the user is active

- Active lifetime; the number of days between the first and the last post of the user
- Wait time; the number of days passed between the day the user joined the forum and the day the user contributed their first post

- $\quad$ Average post length; the average number of characters in the user's posts 
- $\quad$ Median post length; the median number of characters in the user's posts

- Maximum post length; the number of character's in the user's longest post

Step 2: Contextual Features. Apart from the aforementioned behavioral features we also include features related with the context in which an IP address appears within a post. In particular, we consider the frequency of the words (except stop-words) in the posts. Words that are frequent only in few documents (posts in our case) are more informative than those that appear frequently on a larger corpus [21]. To this end, we use TF-IDF to weight the various words/terms that appear in our data. After calculating the frequency and the corresponding weights of each word in the dataset we end up with more than 10,000 features/terms. Hence, in the next step we select discriminative features by extracting latent features.

We begin by performing feature selection in order to identify the most informative features by applying the information gain framework [25]. Furthermore, in order to avoid overfitting we pick a random subset of posts from the whole dataset and select the highest ranked features based on Information Gain score. In this way, a subset of discriminative keywords, 100 in our model, are selected. It turns out that each user uses only a small number of those words, resulting in a sparse dataset which we wish to exploit in our model.

Step 3: Identifying latent feature sets. We also like to leverage latent similarities of different posts in some of the dimensions spanned by post features and behavioral features for the writer of the post. Essentially, we seek to identify groups of highly similar posts under a small number of features, which does not necessarily span the full set of features. The reason why we wish to pinpoint a subset of the features instead of the entire set is because this way we are able to detect subtle patterns that may go undetected if we require post similarity across all the features. We call those sets of feastures latent feature sets . To this end, we apply a soft co-clustering method, Sparse Matrix Regression (SMR) [19], to exploit the sparsity and extract latent features of the post containing IP addresses. Given a matrix $\mathbf{X}$ of posts $\times$ features, its soft co-clustering via SMR can be posed as the following optimization problem:

$$
\min _{\lambda \sum_{j, r}\left|\mathbf{b}_{r}(j)\right|}\left\|0, \mathbf{b}_{r} \geq 0\right\| \mathbf{X}-\sum_{r}^{R} \mathbf{a}_{r} \mathbf{b}_{r}^{T} \|_{F}^{2}+\lambda \sum_{i, r}\left|\mathbf{a}_{r}(i)\right|+
$$

where $\mathbf{a}_{r}$ and $\mathbf{b}_{r}$ are vectors that "describe" co-cluster $r$, which we explain below. Each $\mathbf{a}_{r}$ is a vector with as many dimensions as posts. Each value $\mathbf{a}_{r}(i)$ expresses whether post $i$ is affiliated with co-cluster $r$. Similarly, $\mathbf{b}_{r}$ is a vector with as many dimensions as features, and $\mathbf{b}_{r}(j)$ expresses whether feature $j$ is affiliated with with co-cluster $r$. Parameter $\lambda$ controls how sparse the co-cluster assignments are, effectively controlling the co-cluster size. As we increase $\lambda$ we get sparser results, hence cleaner co-clustering assignments. We tune $\lambda$ via trial-and-error so that we obtain clean but non-empty coclusters, and we select $\lambda=0.01$ in our case.

Step 4: Training the model. We subsequently train a number of classifiers using the selected features based on a matrix. In particular, we examine (a) a Naive Bayes classifier, (b) a K-Nearest Neighbor classifier and (c) a logistic regression classifier. Our 10-fold cross validation indicates that the Logistic regression classifier outperforms $\mathrm{kNN}$ and Naive Bayse, achieving high accuracy, precision and recall (see Table III).

Determining feature sets. We investigate the effect of selecting different feature sets in classifying IP addresses in forums. To this end, we investigate three subsets of the features discussed earlier.

a. Words-Frequency is the normalized frequency of the most informative words that appear in a post as discussed in Step 2.

b. Combined is the set of features which consists of the combination of the words frequency features, defined above, and user behaviour features, which are extracted in Step 1. In other words, it is the union of the features in Step 1 and Step 2 .

c. Co-Clustered is the latent set of features extracted in Step 3 by applying the co-clustering approach on the Combined features set.

We evaluate these three sets of features on their ability to enable the classification. In more detail, we use these features with a classifier to assess their effectiveness by computing the accuracy of the classifier to identify malicious IP addresses. According to the results which are shown in Figure 4 the Co-Clustered features set exhibits higher accuracy by $4.1 \%$ compared to Words-Frequency. On the other hand, although the Combined features do not increase the accuracy compared to the Words-Frequency, the co-clustering method does. It extracts the latent features from the Combined features set and outperforms Words-Frequency and Combined in identifying malicious IP addresses.

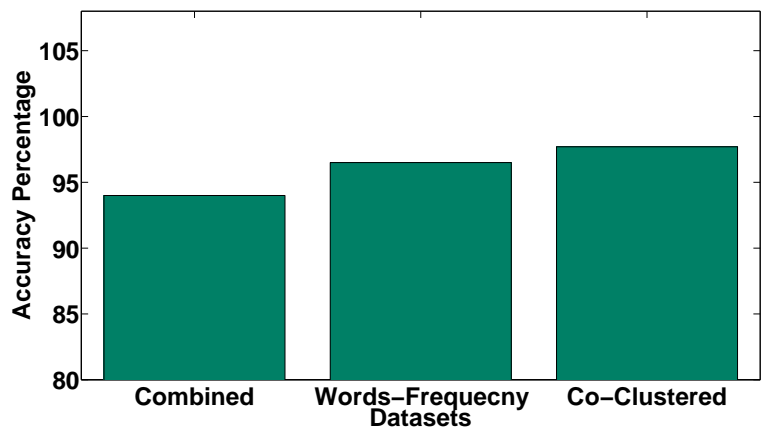

Fig. 4: Accuracy of different feature sets in Wilders Security forum to detect malicious IP

\section{A. Applying InferIP on the forums}

Having established the statistical confidence of our classifier, we apply it on the posts of the forums except the ones that we used in our groundtruth. We use the logistic regression classifier as it exhibits the best performance.

Applying InferIP on the forums shows that there is a wealth of information that we can extract from security forums in two aspects of the quantity and time of detecting malicious IP against VirusTotal.

a. Detecting more IP addresses. With InferIP, we find an additional 670 malicious IP addresses in Wilders Security, and 
TABLE III: Selecting a classifier: overall accuracy.

\begin{tabular}{c|c|c|c}
\hline Forum & Naive Bayes & 3NN & Logistic regression \\
\hline Wilders Security & $91.9 \%$ & $87.1 \%$ & $94.8 \%$ \\
Offensive Comm. & $84.1 \%$ & $83.2 \%$ & $86.5 \%$ \\
Ashiyane & $85.1 \%$ & $82.3 \%$ & $94 \%$ \\
\hline
\end{tabular}

TABLE IV: InferIP evaluation: 10-fold cross validation evaluation (using Logistic Regression).

\begin{tabular}{c|c|c|c|c}
\hline Forum & Instances & Precision & Recall & ROC Area \\
\hline Wilders Security & 362 & 0.9 & 0.94 & 0.96 \\
Offensive Comm. & 342 & 0.88 & 0.85 & 0.91 \\
Ashiyane & 446 & 0.9 & 0.92 & 0.92 \\
\hline
\end{tabular}

617 in Offensive Community 806 in Ashiyane (see Table I). In other words, InferIP enables us to find three times additional malicious IP addresses in total compared to the IP addresses found on VirusTotal. It is interesting to observe that this factor varies among our three sites. For Ashiyane, our method finds roughly 6 times additional malicious IP addresses. With a precision of roughly around $90 \%$ and considering small amount of False Positive rate, our method can add a significant number of malicious IP addresses to a blacklist. Using the limited manual inspection, we confirm that the precision of the method on out of sample data is in the order of $88 \%$.

b. Detecting malicious IP addresses earlier: more than half IPs, at least 3 months earlier Here we focus on the malicious IP addresses that are jointly identified by our method and VirusTotal and compare the time that they were reported in each source, and show the results in Table $[\mathrm{V}]$ for 3,6 and 12 months difference in time. We compare jointly detected IP addresses with InferIP and VirusTotal in terms of time that the IP addresses were mentioned in posts and the time they were reported on VirusTotal. We see that on average $62 \%$ of the malicious IP addresses with InferIP could be identified at least 3 months earlier than VirusTotal. We can see that with InferIP, we find $53 \%, 71 \%$ and $62 \%$ of these IP addresses in Wilders Security, Offensive Community and Ashiyane respectively at least 3 months earlier than in VirusTotal. We also identify $39 \%$ and $24 \%$ of the malicious IP addresses respectively at least 6 and 12 months earlier with InferIP.

Additional stress-testing of our accuracy: In order to assess the performance of our approach, we randomly picked 10 percent of the labeled data with InferIP method and annotated them manually by human annotators. The calculated accuracy on the sampled data shows more than $85 \%$ accuracy on average over all datasets which is close but somewhat lower than the reported accuracy in the Table III.

Contributing Users. Who are the users that report malicious IP addresses? We want to understand and ideally, develop a profile for these users, which we will refer to as Contributing users. We start by considering the number of post these users post on the forums.

The majority of IP reporting is done by highly active (more than 10 posts overall) in Ashiyane. In Figure 5] we show the cumulative complementary distribution function for the number of posts per Contributing user for Ashiyane.
TABLE V: Timely comparison between jointly detected malicious IP addresses in InferIP and VirusTotal. Reported percentage of malicious IP Addresses which InferIP detected earlier than VirusTotal

\begin{tabular}{|c|c|c|c|}
\hline & \multicolumn{3}{|c|}{ At least X months earlier } \\
\hline Dataset & 3 & 6 & 12 \\
\hline Wilders Security & $53 \%$ & $23 \%$ & $14 \%$ \\
Offensive Community & $71 \%$ & $46 \%$ & $21 \%$ \\
Ashiyane & $62 \%$ & $49 \%$ & $37 \%$ \\
\hline Average (across forums) & $62 \%$ & $39 \%$ & $24 \%$ \\
\hline
\end{tabular}

More than $72 \%$ of the Contributing users post more than 10 posts overall, which we consider as high engagement given the distribution of posting that we saw in the previous section. Therefore, in Ashiyane, Contributing users are contributing significantly in reporting malicious IP addresses. Intrigued, we examined further and found that, among them, there are two users who have more than 1000 posts, 1058 and 2780 to be exact, and whose user-names are "Classic" and "Crisis". On the other side of the spectrum, $2.4 \%$ of Contributing users have posted a single post in the forum, and in that post they reported a malicious IP address.

The majority of IP reporting is done by less active users (less than 10 posts overall) in Offensive Community. In Figure 5, we show the cumulative complementary distribution function for the number of posts per Contributing user for Offensive Community. Unlike Ashiyane, here $65 \%$ of the Contributing users have less than 10 posts overall. Going into more detail, roughly $12 \%$ of the Contributing users have a single post overall, while $26 \%$ of them have only two overall posts. The same behavior is observed in Wilders Security which is shown in Figure 5

Overall, there does not seem to be an obvious pattern between number of total posts and number of malicious IPs reported among Contributing users.

\section{B. Case-study: from reported malicious IPs to a DDoS attack}

We show that mining the forums could actually provide information about real events. We identify a link between a malicious IP address that our method detected with an actual DDoS attack. 


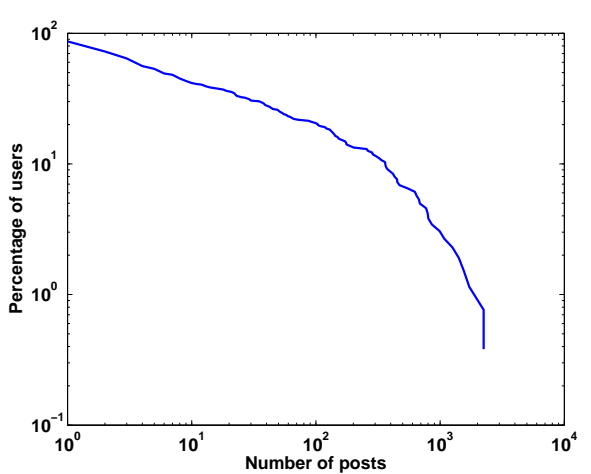

(a) Wilders Security

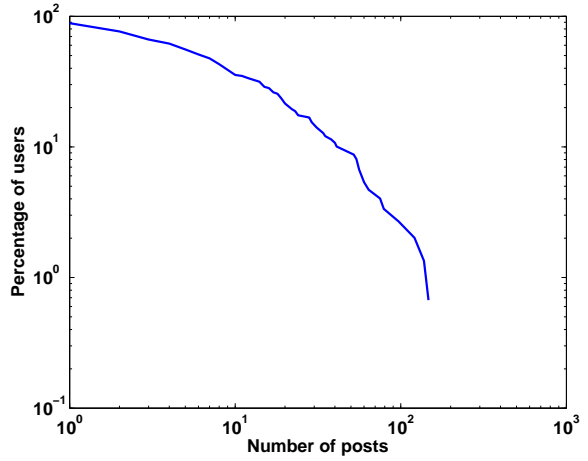

(b) Offensive Community

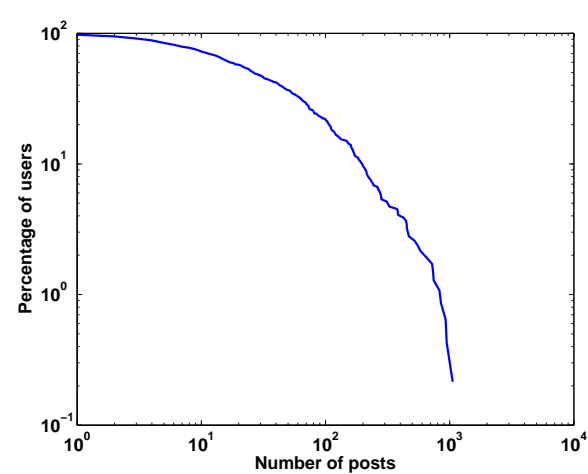

(c) Ashiyane

Fig. 5: CCDF of the number of overall posts per Contributing users (who report malicious IPs) in log-log scale.

We conducted the following analysis. We plot the timeseries of the number of posts containing malicious IP addresses in Wilders Security from 2012 to 2013 found by InferIP. We show the time-series in Figure 6 . We observe some spikes on these time-series, which we further analyze. One of the spikes was in September 2012, and it reports a set of malicious IP addresses that were involved in an DDoS attack that month. That same thread continued being active, and in December of 2012 , it was reported in that thread that attack was caused by Nitol Botnet due to a Microsoft's vulnerability [3].

We argue that this case-study points to additional layers of functionality that can be built upon our method, that can provide a semi-automated way to extract richer information beyond just reporting malicious IP addresses.

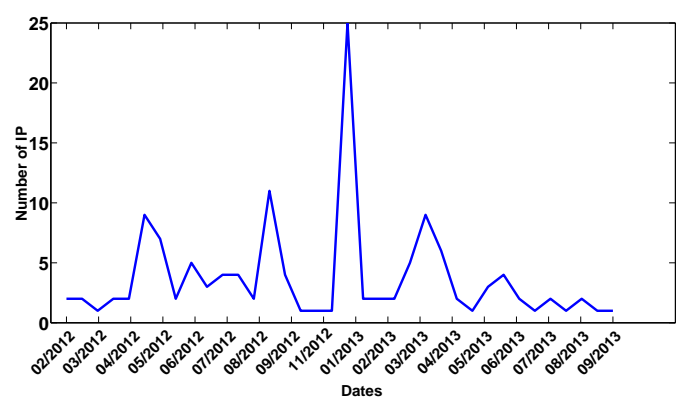

Fig. 6: Time-series of the number of posts containing malicious IP reported in each month for Wilders Security.

\section{Discussion and limitations}

Although our method exhibits pretty good accuracy overall, we attempt to understand its limitations and detect the source of misclassifications.

Limited text in the post: The words in the post provide significant evidence for the classification. In some cases, some posts are very sparse in their text, which makes the classification of the included IP address harder. We consider these kinds of posts a significant contributor to misclassifications.

Characterization at the post level: In our method, we classify an IP address by using features at the level of a post. Recall that roughly $86 \%$ of all posts across all forums has a single IP per post as shown in Figure 3. In other words, having more than one IP address per post is already not very common. Furthermore, even more rarely, we have seen a few cases, where a post contains both a benign and a malicious IP address. As our method is currently set-up, this will lead to errors in the classification. A straightforward solution is to consider examining the text surrounding each IP address within the post.

\section{SPATIOTEMPORAL ANALYSis}

In this section, we discuss the spatiotemporal features of the malicious IP addresses identified in security forums in Section [II]

\section{A. Temporal analysis}

The key question from a temporal point of view is if the number of reported malicious IP addresses increases or decreases over time.

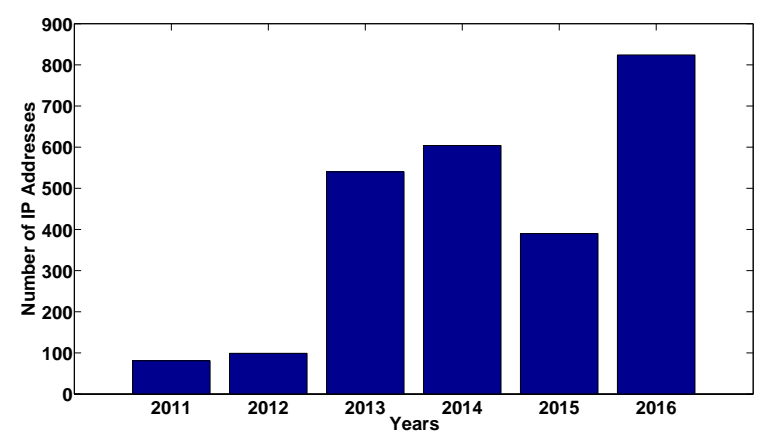

Fig. 7: Increasing trend: Malicious IP addresses reported on the forums each year.

The number of reported malicious IP addresses has increased by a factor 8 in $\mathbf{4}$ four years. In Figure 7 we plot the number of reported malicious IPs found by our method across all three forums between 2011-2016. We find that the number increased by a factor of 8: from roughly 100 to roughly 800. In spite of some decreases in years 2011, 2012 and 2015, it has a clear increasing trend. 


\section{B. Spatial analysis}

We study the geo-location of the identified IP addresses from Section [III] We utilize GeoLite database [2], which can show us the country and continent of an IP address. Here we focus on continents of the IP addresses location.

A natural question to ask is whether the geographical distribution of the malicious addresses differs between VirusTotal and InferIP. We investigate this in detail below.

VirusTotal: North America hosts the majority of the reported malicious IP addresses. We plot the percentage of the distribution of the IP addresses extracted from VirusTotal across continents in Figure 8 (a) between 2011-2016. We observe that the majority of the malicious IP addresses are located in the North America continent. There are two exception in 2013 and 2016 when Asia and Europe respectively contain most of the malicious IP addresses. Overall, Table VI] shows the geo-graphical distribution over all the years: North America, Asia and Europe are the three most active continents in that order.

InferIP: North America dominates again, but South America and Africa have non-trivial contributions. We plot the percentage of the distribution of the IP addresses extracted form InferIP across continents in Figure 8 (b) between 20112016. We observe that North America hosts the majority of the reported malicious IP addresses again, but we find a more diverse global activity compared to what we observed in VirusTotal. For example, we can see that in years 2013, 2014, and 2016: (a) Asia has the majority of the malicious IP addresses, and (b) South America and Africa have a considerable percentage of malicious IP addresses. However, when seen across all years, the geographical distributions of the IPs in InferIP and VirusTotal quite similar: North America, Asia and Europe have the majority of the malicious IPs detected by InferIP similarly to those of VirusTotal. In Figure 9 , we plot the geographical distribution of malicious IPs per continent across all years and all forums for InferIP and VirusTotal, while the exact numbers are shown in Table VI] Qualitatively the distributions look relatively similar, especially in the order of significance of the continents, but at the same, we can see that South America and Africa have a larger percentage of IP addresses in InferIP compared to those in VirusTotal.

\section{RELATED WORK}

We briefly discuss three categories of relevant research.

a. Analyzing structured security sources. There is a long line of research studying the ontology of cyber security and the automatic extraction of information from structured security documents. Iannacone et al.[16] developed a schema for extracting relevant concepts from various types of structured data sources. In another work, Blanco et al. [9] proposed methods to detect anomalies on the extracted ontology and network flow graph. Moreover, Bridges et al.[10] proposed a method to do entity labeling on structured data by utilizing neural networks. These work are complementary to ours as we focus on unstructured data, which poses different challenges.

b. Analyzing online security forums. Recently security forums have been the focus of various studies that showcase the usefulness of the information present in security forums.
For example, Motoyama et al. [18] present a comprehensive statistical analysis in underground forums. Others studies focus on the users' classification or the discovery of the relationships between the forum's members [26], [7]. Extracting different discussion topic in the forums and classifying the language of the codes posted in the forum has been done in [23]. Contrary to these studies, our work emphasizes on the development of automated systems that actually exploit the wealth of information in these security forums in order to enhance security. Similar to detecting malicious users on commenting platforms has been done on [17]. A recent work analyzes security forums to identify and geo-locate Canadian IP addresses focusing on spam and phishing [13] and in another work, Portnoff et al. [20] studies the exchange of malicious services and tools and studies their prices on the security forums.

c. Analyzing blogs and social networks. There has been a plethora of studies on blogs and social media, but their goals are typical not related to extracting security information. [11], [8], [24]. The studies range from modeling user behavior [12], [22] to inferring information about the user (demographics, preferences, mental state), and to modeling the information propagation on online forums. Although interesting, the focus of these studies are significantly different from our goal here.

\section{CONCLUSION}

The take away message from our work is that there seems to be a wealth of useful information in security forums. The challenge is that the information is unstructured and we need novel methods to extract it. In this direction, a key insight of our work is that using behavioral and text-based features can provide promising results.

In support of this assertion, we develop a systematic method to extract malicious IP addresses reported in security forums. We utilize both behavioral, as well as textual features and show that we can detect malicious IP addresses with

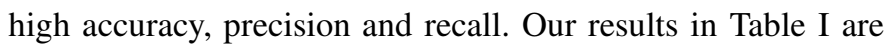
promising.

We then apply InferIP to all the posts we have collected. Although are classification is not perfect, our relatively high precision (hovering around $90 \%$ in Table IV] provides sufficient confidence in our results. We find three times as many additional malicious IP addresses as the original malicious IP addresses identified by VirusTotal. Furthermore, even for the jointly discovered IP addresses, at least $53 \%$ of the IP addresses detected at least 3 months earlier than VirusTotal. The key message from our spatiotemporal analysis is that the number of reported malicious IP addresses is increasing over time.

In the future, we plan to extend our work by extracting other types of security information. Our first goal is to detect malicious URLs mentioned in the forums. Our second and more ambitious goal is to identify the emergence of new malware, threats, and possibly attacks, which we expect to see associated with large numbers of panic-filled or helprequesting posts. Our final goal is to identify malicious users, since interestingly, some users seem to be promoting and selling hacking tools in these forums. 


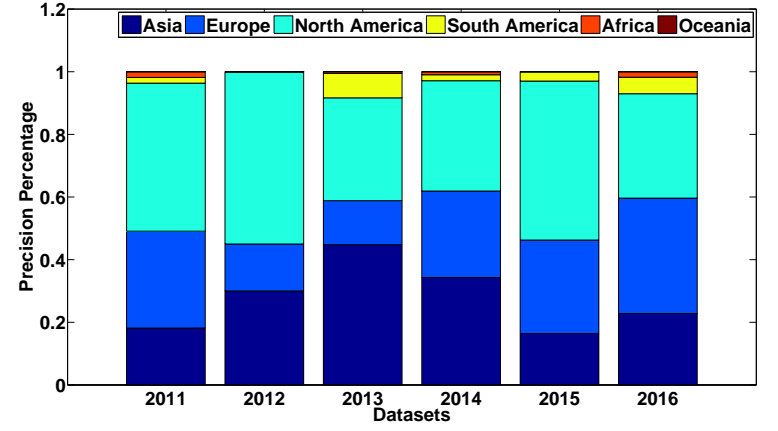

(a) VirusTotal

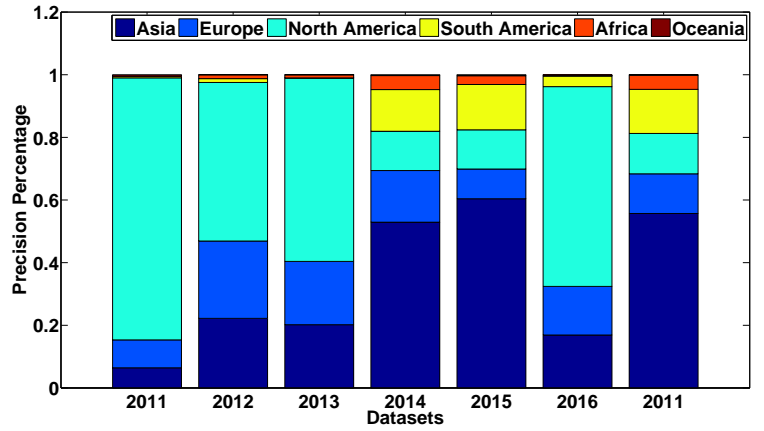

(b)InferIP

Fig. 8: SpatioTemporal distribution of malicious IP addresses detected by InferIP and VT .

TABLE VI: Percentage of distribution of IP addresses across continents over all the years.

\begin{tabular}{|c|c|c|c|c|c|c|}
\hline & North America & Asia & Europe & South America & Africa & Oceania \\
\hline InferIP & $\mathbf{4 6 . 7}$ & 32.5 & 13.5 & 5.2 & 1.6 & 0.5 \\
VirusTotal & $\mathbf{5 0}$ & 26.5 & 20.4 & 2.4 & 0.6 & 0.17 \\
\hline
\end{tabular}

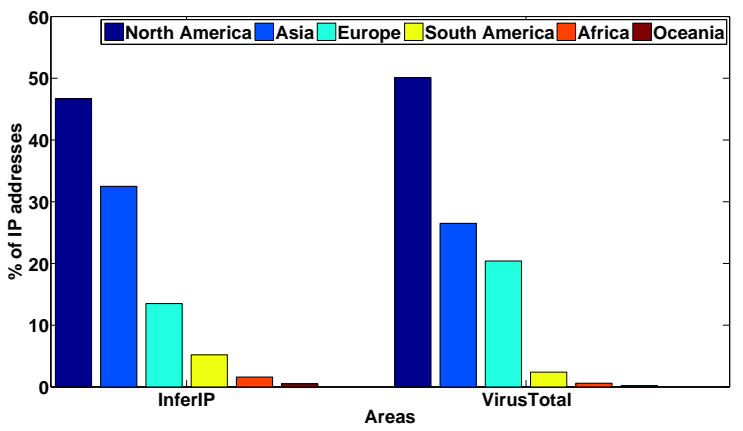

Fig. 9: The percentage distribution of malicious IP Addresses in each continent across all three forums for InferIP and VirusTotal.

\section{ACKNOWLEDGMENTS}

This material is based upon work supported by an Adobe Data Science Research Faculty Award, and DHS ST Cyber Security (DDoSD) HSHQDC-14-R-B00017 grant. Any opinions, findings, and conclusions or recommendations expressed in this material are those of the author(s) and do not necessarily reflect the views of the funding institutions.

\section{REFERENCES}

[1] Ashiyane. http://www.ashiyane.org/forums/

[2] Geolite. http://dev.maxmind.com/geoip/legacy/geolite/

[3] Nitol-botnet. https://threatpost.com/tag/nitol-botnet/

[4] Offensive community. http://www.offensivecommunity.net

[5] Virustotal. http://www.virustotal.com

[6] Wilders security. http://www.wilderssecurity.com

[7] A. Abbasi, W. Li, V. Benjamin, S. Hu, and H. Chen. Descriptive analytics: Examining expert hackers in web forums. In 2014 IEEE Joint Intelligence and Security Informatics Conference, pages 56-63, Sept 2014.

[8] T. Althoff, P. Jindal, and J. Leskovec. Online actions with offline impact: How online social networks influence online and offline user behavior. In Proceedings of the Tenth ACM International Conference on Web
Search and Data Mining, WSDM '17, pages 537-546, New York, NY, USA, 2017. ACM.

[9] C. Blanco, J. Lasheras, R. Valencia-García, E. Fernández-Medina, A. Toval, and M. Piattini. A systematic review and comparison of security ontologies. In 2008 Third International Conference on Availability, Reliability and Security, pages 813-820, March 2008.

[10] R. A. Bridges, C. L. Jones, M. D. Iannacone, and J. R. Goodall. Automatic labeling for entity extraction in cyber security. CoRR, abs/1308.4941, 2013.

[11] J. Cheng, M. Bernstein, C. Danescu-Niculescu-Mizil, and J. Leskovec. Anyone Can Become a Troll: Causes of Trolling Behavior in Online Discussions. ArXiv e-prints, Feb. 2017.

[12] P. Devineni, D. Koutra, M. Faloutsos, and C. Faloutsos. If walls could talk: Patterns and anomalies in facebook wallposts. In Proceedings of the 2015 IEEE/ACM International Conference on Advances in Social Networks Analysis and Mining 2015, ASONAM '15, pages 367-374, New York, NY, USA, 2015. ACM.

[13] R. Frank, M. Macdonald, and B. Monk. Location, location, location: Mapping potential canadian targets in online hacker discussion forums. EISIC '16, 2016.

[14] J. Gharibshah, T. C. Li, M. S. Vanrell, A. Castro, K. Pelechrinis, E. E Papalexakis, and M. Faloutsos. Inferip: Extracting actionable information from security discussion forums. In Proceedings of the 2017 IEEE/ACM International Conference on Advances in Social Networks Analysis and Mining 2017, ASONAM '17, pages 301-304, New York, NY, USA, 2017. ACM.

[15] H. Hang, A. Bashir, M. Faloutsos, C. Faloutsos, and T. Dumitras "Infect-me-not": A user-centric and site-centric study of web-based malware. In IFIP Networking, pages 234-242, May 2016.

[16] M. Iannacone, S. Bohn, G. Nakamura, J. Gerth, K. Huffer, R. Bridges, E. Ferragut, and J. Goodall. Developing an ontology for cyber security knowledge graphs. In Proceedings of the 10th Annual Cyber and Information Security Research Conference, CISR '15, pages 12:1-12:4, New York, NY, USA, 2015. ACM.

[17] T. C. Li, J. Gharibshah, E. E. Papalexakis, and M. Faloutsos. Trollspot: Detecting misbehavior in commenting platforms. In Proceedings of the 2017 IEEE/ACM International Conference on Advances in Social Networks Analysis and Mining 2017, ASONAM '17, 2017.

[18] M. Motoyama, D. McCoy, K. Levchenko, S. Savage, and G. M. Voelker. An analysis of underground forums. In Proceedings of the 2011 ACM SIGCOMM Conference on Internet Measurement Conference, IMC '11, pages 71-80, New York, NY, USA, 2011. ACM.

[19] E. E. Papalexakis, N. D. Sidiropoulos, and R. Bro. From k-means to higher-way co-clustering: Multilinear decomposition with sparse latent factors. IEEE transactions on signal processing, 61(2):493-506, 2013. 
[20] R. S. Portnoff, S. Afroz, G. Durrett, J. K. Kummerfeld, T. BergKirkpatrick, D. McCoy, K. Levchenko, and V. Paxson. Tools for automated analysis of cybercriminal markets. WWW'17, 2017.

[21] J. Ramos. Using TF-IDF to determine word relevance in document queries. In Instructional Conference on Machine Learning, 2003.

[22] R. Rawassizadeh, E. Momeni, C. Dobbins, J. Gharibshah, and M. Pazzani. Scalable daily human behavioral pattern mining from multivariate temporal data. IEEE Transactions on Knowledge and Data Engineering, 28(11):3098-3112, Nov 2016.

[23] S. Samtani, R. Chinn, and H. Chen. Exploring hacker assets in underground forums. In IEEE International Conference on Intelligence and Security Informatics (ISI), pages 31-36, May 2015.

[24] J. Ugander, B. Karrer, L. Backstrom, and C. Marlow. The anatomy of the facebook social graph. CoRR, abs/1111.4503, 2011.

[25] Y. Yang and J. O. Pedersen. A comparative study on feature selection in text categorization. In Proceedings of the Fourteenth International Conference on Machine Learning, ICML '97, pages 412-420, San Francisco, CA, USA, 1997. Morgan Kaufmann Publishers Inc.

[26] X. Zhang, A. Tsang, W. T. Yue, and M. Chau. The classification of hackers by knowledge exchange behaviors. Information Systems Frontiers, 17(6):1239-1251, Dec. 2015. 